\title{
High-throughput sequencing reveals the core gut microbiota of the mud crab (Scylla paramamosain) in different coastal regions of southern China
}

\author{
Hongling Wei ${ }^{1}$, Huan Wang ${ }^{1,2^{*}}$, Lei Tang ${ }^{1}$, Changkao Mu ${ }^{1,2}$, Chunyu Ye ${ }^{3}$, Lizhi Chen ${ }^{4}$ and Chunlin Wang ${ }^{1,2^{*}}$ (D)
}

\begin{abstract}
Background: Scylla paramamosain is a commercially important mud crab. The microbiota is a community that inhabits the crab intestine, and is important for physiological functional and host health.

Results: Proteobacteria, Firmicutes, Bacteroidetes, Tenericutes, Spirochaetae and Fusobacteria were the dominant phyla of the 36 representative phyla. Eleven genera of the 820 representative genera were considered as core gut microbiota and were distributed in the five dominant phyla. The core genus of the Proteobacteria included Arcobacter, Photobacterium, Vibrio, Shewanella and Desulfovibrio. The other four phyla contained one or two genera. Male and female crab samples had two different core genera, (male samples: Psychrilyobacter \& Lactococcus; female samples: Clostridium_sensu_stricto_11 and Candidatus_Bacilloplasma).

Conclusions: This is the first time core intestinal microbiota have been identified in crab from nine coastal regions of southern China. This study provides sequencing data related to the gut microbiota of S. paramamosain, and may contribute to probiotic development for S. paramamosain aquaculture industries.
\end{abstract}

Keywords: Scylla paramamosain, Core gut microbiota, Illumina MiSeq sequencing, 16S rRNA

\section{Background}

Scylla paramamosain is a commercially important mud crab distributed along the coasts of southern China and other Indo-Pacific countries [1-4]. Mud crab production reached 231,467 tons in 2017 in China [5]. Currently, thanks to its richness, rapid growth, and high market value, the species is important in both fisheries and aquaculture in southern China [6-8].

The microbiota inhabits the intestine which is an important physiological functional organ in S. paramamosain, and is closely related to host health $[9,10]$. Much research in humans has shown that the gut microbiota plays basic roles in nutrient absorption and immune function, which is beneficial to host health $[11,12]$. Some pathological conditions, such as, inflammatory bowel disease [13],

\footnotetext{
* Correspondence: wanghuan1@nbu.edu.cn; wangchunlin@nbu.edu.cn ${ }^{1}$ School of Marine Science, Ningbo University, Ningbo 315211, Zhejiang, China

Full list of author information is available at the end of the article
}

liver cirrhosis [14], cancer [15], obesity [16], and Type 1 Diabetes [17] appear to be caused by disruption to its normal balance. Research has shown that the gut microbiota are widely involved in organ development, nutrition, immunity and crustacean diseases [18-21]. Other gut microbiome research has shown that the health, eating habits and crustacean habitats are key to the formation of a symbiotic gut bacteria model $[22,23]$. Although a close relationship between the crab and its gut microbiota is increasingly accepted, limited data are available on the gut microbiota of S. paramamosain from Southern Chinese coasts. As part of aquaculture development, it is crucial to develop better probiotics to facilitate $S$. paramamosain industries, by unraveling gut microbial composition.

In this study, Illumina MiSeq sequencing of 16S rRNA was used to identify gut microbial composition in $S$. paramamosain. Samples from southern Chinese coasts were compared to characterize core gut microbiota.

(c) The Author(s). 2019 Open Access This article is distributed under the terms of the Creative Commons Attribution 4.0 International License (http://creativecommons.org/licenses/by/4.0/), which permits unrestricted use, distribution, and 


\section{Results}

\section{Data summary}

After filtering low-quality reads, trimming the longer homopolymer runs, adapters, barcodes and primers, and rarefying datasets, 21,993 (sample YJ-M) to 28,377 (sample XP-F) valid contigs were collected from each region, resulting in a total of 472,782 valid contigs from the nine regions. All valid contigs were delineated into OTUs using 97\% sequence similarity thresholds, consistent with other studies performing deep sequencing methods [24]. A total of 2552 OTUs were obtained. Each region sample contained 87 (sample XP-F) to 755 (sample HLM) OTUs, which differed no significantly in most of the male crabs samples than the female crabs samples (Table 1). Nevertheless, HL and ST crabs generated significant differences between female and male samples (HL: $P=0.0041$, ST: $P=0.0037$ ).

The characterization of bacterial community richness, diversity and sequencing depth was performed using the alpha diversity index (Table 1). The Chao1 indices, which ranged from $129 \pm 57.67$ to $858 \pm 109.83$, were used to determine bacterial community richness in S. paramamosain. There were no differences in Shannon and Simpson indices. The Good's coverage estimator of the samples ranged from 0.990 to 0.998 (Table 1), indicating that sequencing depths covered all species in samples. Meanwhile, the sparse curve reaches the saturation platform (Additional file 1: Figure S1A), manifesting that the sequencing depth is large enough to obtain a stable and unbiased estimate of species richness. In addition, the specaccum accumulation curves tend to gradually, indicating that the sample size is sufficient to reflect the abundance of the community richness, the results reflect the rate of increase in new species observed as the sample size continues to increase during the overall sampling of the sample. The number of OTUs increased rapidly from 1 to 54 and began to level off at the end of our sampling, indicating that bacterial diversity was largely saturated (Additional file 1: Figure S1B).

\section{Composition of microbial communities in $S$. paramamosain}

At the taxonomic level, six different patterns of intestinal microbial composition were distinguished. As shown (Additional file 1: Table S1), the number of taxonomic units detected in each region were present. The pattern of gut microbial composition in male samples was greater than female samples (Additional file 1: Table S1). Additional file 1: Figure S2-S4 show microbial community composition at Class, Order, and Family levels.

We found 36 different phyla (Additional file 1: Table S1) in all samples. There were differences between male and female samples (30 phyla in female samples and 35 phyla in male samples). Figure 1a and Additional file 1: Table S2 show the top 15 highly abundant phyla. Tenericutes, Proteobacteria, Bacteroidetes, Firmicutes and Fusobacteria were identified in all samples, another ten phyla were only detected in one or several samples, including Spirochaetae, Actinobacteria, Acidobacteria, Gemmatimonadetes, CKC4, Deferribacteres, Cyanobacteria,

Table 1 Overview of sequencing data and alpha-diversity of samples from the nine coastal regions of southern China

\begin{tabular}{|c|c|c|c|c|c|c|}
\hline Group & Valid contigs & OTU & Shannon & Simpson & Chao 1 & Good's coverage \\
\hline$\overline{H L-F}$ & $26,157 \pm 1969$ & $215 \pm 57$ & $3.48 \pm 0.216$ & $0.84 \pm 0.030$ & $338 \pm 125.87$ & $0.995 \pm 0.0016$ \\
\hline HL-M & $26,168 \pm 2337$ & $755 \pm 148$ & $5.44 \pm 0.993$ & $0.91 \pm 0.021$ & $858 \pm 109.83$ & $0.988 \pm 0.0008$ \\
\hline HP-F & $27,904 \pm 860$ & $154 \pm 53$ & $3.42 \pm 0.495$ & $0.80 \pm 0.040$ & $189 \pm 52.33$ & $0.997 \pm 0.0008$ \\
\hline HP-M & $26,118 \pm 2418$ & $453 \pm 280$ & $4.29 \pm 1.123$ & $0.88 \pm 0.070$ & $527 \pm 317.45$ & $0.992 \pm 0.0047$ \\
\hline SM-F & $28,219 \pm 821$ & $286 \pm 176$ & $4.57 \pm 2.047$ & $0.89 \pm 0.080$ & $318 \pm 150.82$ & $0.997 \pm 0.0014$ \\
\hline SM-M & $27,103 \pm 1061$ & $270 \pm 113$ & $3.41 \pm 0.367$ & $0.79 \pm 0.066$ & $353 \pm 146.69$ & $0.994 \pm 0.0025$ \\
\hline RA-F & $27,495 \pm 1216$ & $318 \pm 193$ & $4.79 \pm 1.872$ & $0.90 \pm 0.079$ & $385 \pm 133.53$ & $0.996 \pm 0.0015$ \\
\hline RA-M & $25,757 \pm 2074$ & $179 \pm 43$ & $3.85 \pm 0.594$ & $0.86 \pm 0.075$ & $213 \pm 65.40$ & $0.997 \pm 0.0011$ \\
\hline ST-F & $26,952 \pm 1164$ & $174 \pm 66$ & $3.09 \pm 1.118$ & $0.75 \pm 0.143$ & $248 \pm 107.24$ & $0.996 \pm 0.0015$ \\
\hline ST-M & $27,729 \pm 2215$ & $429 \pm 30$ & $4.84 \pm 1.043$ & $0.92 \pm 0.045$ & $596 \pm 91.95$ & $0.991 \pm 0.0022$ \\
\hline TS-F & $22,555 \pm 3723$ & $530 \pm 242$ & $3.55 \pm 0.896$ & $0.76 \pm 0.075$ & $617 \pm 269.36$ & $0.990 \pm 0.0044$ \\
\hline TS-M & $26,197 \pm 1560$ & $186 \pm 97$ & $3.55 \pm 0.579$ & $0.84 \pm 0.071$ & $266 \pm 170.60$ & $0.996 \pm 0.0030$ \\
\hline$X P-F$ & $28,377 \pm 698$ & $87 \pm 14$ & $2.47 \pm 0.471$ & $0.70 \pm 0.068$ & $129 \pm 57.67$ & $0.998 \pm 0.0006$ \\
\hline XP-M & $26,811 \pm 1415$ & $92 \pm 43$ & $2.43 \pm 0.984$ & $0.66 \pm 0.217$ & $153 \pm 103.13$ & $0.998 \pm 0.0015$ \\
\hline YJ-F & $26,447 \pm 657$ & $594 \pm 368$ & $5.12 \pm 2.241$ & $0.89 \pm 0.086$ & $695 \pm 394.79$ & $0.991 \pm 0.0054$ \\
\hline YJ-M & $21,993 \pm 5211$ & $378 \pm 80$ & $5.52 \pm 1.623$ & $0.92 \pm 0.077$ & $394 \pm 70.29$ & $0.998 \pm 0.0016$ \\
\hline$Y X-F$ & $25,662 \pm 2052$ & $219 \pm 186$ & $4.74 \pm 1.923$ & $0.91 \pm 0.066$ & $242 \pm 167.04$ & $0.998 \pm 0.0004$ \\
\hline YX-M & $25,138 \pm 6631$ & $480 \pm 8$ & $6.98 \pm 0.149$ & $0.98 \pm 0.003$ & $487 \pm 15.34$ & $0.998 \pm 0.0004$ \\
\hline
\end{tabular}



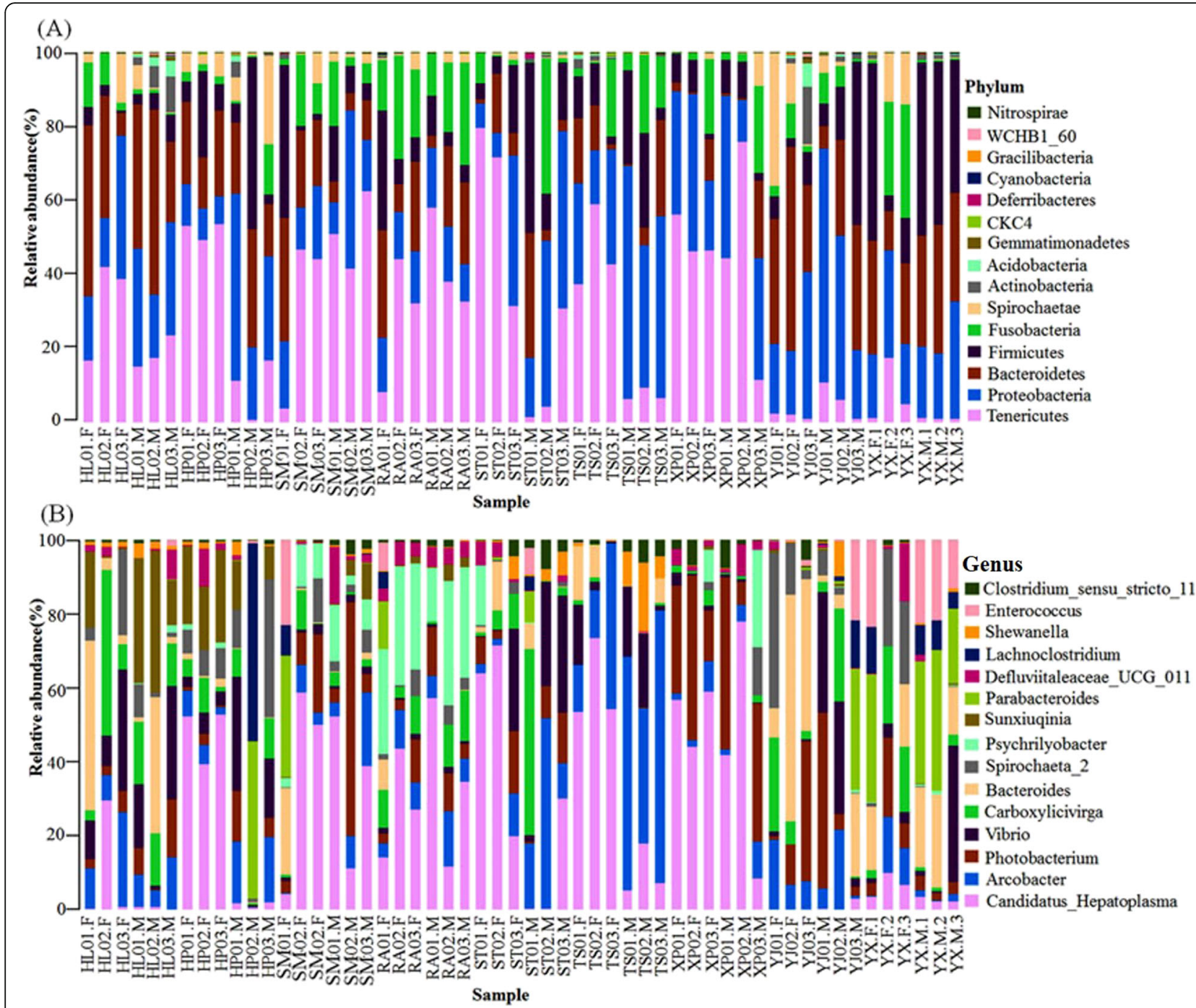

Fig. 1 The 15 most abundant phyla and genera. a Bar-plots showing the abundance and distribution of the 15 most abundant phyla. b Bar-plots showing the abundance and distribution of the 15 most abundant genera

Gracilibacteria, WCHB1_60 and Nitrospirae. Proteobacteria levels were significantly different between female and male samples $(P=0.0085)$. Tenericute levels were significantly different between female and male samples $(P=0.0346)$. No significant differences were found in the other thirteen phyla.

At the genus level, sequences from samples represented 820 genera (Additional file 1: Table S1). Genera in male samples numbered 45 more than in female samples (711 genera in female samples and 756 genera in male samples). The top 15 genera are listed in Fig. $1 \mathrm{~b}$ and Additional file 1: Table S3; Candidatus_Hepatoplasma, Arcobacter, Photobacterium, Vibrio, Carboxylicivirga, Bacteroides, Spirochaeta_2, Psychrilyobacter, Sunxiuqinia, Parabacteroides, Defluviitaleaceae_UCG_011, Lachnoclostridium, Shewanella, Enterococcus, and Clostridium_sensu_stricto_ 11 . These 15 genera accounted for nearly half or more of the total sequences in the samples. Candidatus_Hepatoplasma and Shewanella abundance differed significantly in female samples when compared to male samples $(P=$ $0.0286, P=0.0291$, respectively). No significant differences were found for the other thirteen genera.

Core gut microbiota at the genus level in $S$.

\section{paramamosain}

A major goal of the study was to determine whether a common core microbiota was shared among all samples. At the genus level, we assigned 11 genera candidates (Table 2), each of these candidates exhibited a frequency of occurrence higher than $90 \%$ over all samples and were treated as core gut microbiota [25, 26]. We analyzed 11 core genera and found that these organisms constituted a phylogenetic core of the genera, accounting for $48.81 \%$ of all sequences (Fig. 2a). These 11 core genera were 
Table 2 The core genera identified in samples

\begin{tabular}{llll}
\hline Phylum & Genus & $\begin{array}{l}\text { Relative } \\
\text { abundance (\%) }\end{array}$ & Range (\%) \\
\hline Tenericutes & $\begin{array}{l}\text { Candidatus_ } \\
\text { Hepatoplasma }\end{array}$ & 16.89 & $0.000-74.325$ \\
Proteobacteria & Arcobacter & 6.89 & $0.058-40.186$ \\
Proteobacteria & Photobacterium & 6.81 & $0.000-42.027$ \\
Proteobacteria & Vibrio & 4.48 & $0.074-22.604$ \\
Bacteroidetes & Carboxylicivirga & 3.94 & $0.004-29.438$ \\
Bacteroidetes & Bacteroides & 3.70 & $0.000-47.259$ \\
Spirochaetae & Spirochaeta_2 & 3.48 & $0.000-35.887$ \\
Proteobacteria & Shewanella & 0.85 & $0.000-7.066$ \\
Firmicutes & Lactobacillus & 0.73 & $0.000-11.112$ \\
Firmicutes & Romboutsia & 0.68 & $0.000-17.830$ \\
Proteobacteria & Desulfovibrio & 0.36 & $0.000-4.543$ \\
\hline
\end{tabular}

distributed among five phyla, and $63.6 \%$ of these genera were in the Proteobacteria and Firmicutes, with the remaining in the Bacteroidetes, Tenericutes and Spirochaetae genera. However, from this study, the relative abundance of these core genera varied greatly across samples (Fig. 2b and Table 2).

We also investigated these intergeneric the co-occurrence patterns of these genera based on Spearman's rank correlations (Fig. 2c). We observed that the genus Candidatus Hepatoplasma was inversely associated with almost every other genera (Spearman's rank correlation coefficients $(\rho)$ ranged from - 0.45 to 0.17 ) and Bacteroides showed relatively strong negative correlations with Photobacterium $(\rho=-0.53)$. Vibrio was positively associated with other genera besides Candidatus_Hepatoplasma, Lactobacillus and Bacteroides (Spearman's rank correlation coefficients $(\rho)$ ranged from -0.27 to 0.68 ). Other genera were positively or inversely correlated with each other to different degrees.

We also found genus level differences, between male and female samples, in the core gut microbiota. Fifteen core genera were distributed between male and female samples, (Tables 3 and 4), and thirteen uniform core genera were distributed between male and female samples (Fig. 3). There were two additional core genera in female samples (Clostridium_sensu_stricto_11 \& Candidatus_Bacilloplasma), and two special core genera in male samples (Psychrilyobacter \& Lactococcus).

\section{Gut microbiota relationships across S. paramamosain, in nine regions}

This study investigated relationships of gut microbial communities in 54 samples using weighted UniFrac PCoA and hierarchical dendrogram analyses (Fig. 4). There were obvious separated and overlapped samples for each of the regions. Composition of the community in the nine regions, had changed. Among these, samples derived from SM, RA, XP and YX were grouped closer than the other five flocks. However, there were differences between male and female samples in community compositions. Samples HP-F, ST-F, TS-F, XP-F, YJ-F and YX-F clustered closer than samples HL-F, SM-F and RA-F. Furthermore, samples HF-M, SM-M, TS-M and YX-M were closer than samples HP-M, RA-M, ST-M, $\mathrm{XP}-\mathrm{M}$ and $\mathrm{YJ}-\mathrm{M}$ in term of community composition (Fig. 5a and b). UPGMA clustering analyses, based on weighted UniFrac distances, also indicated a similarly discriminative structural separation between male and female samples (Fig. 5c, d).

\section{Discussion}

S. paramamosain are usually cultured in brackish, seawater ponds along the coasts of southern China and other Indo-Pacific countries. It is a commercially important mud crab distributed [3, 27]. Breeding of S. paramamosain mainly occurs along the coasts of southern China (Additional file 1: Figure S1), including Zhejiang, Fujian, Guangdong, Guangxi, Hainan province. Although gut microbiota regulates many aspects of digestive function, nutrition, metabolism, fat storage and gut-associated mucosal immunity [28], little is known about gut bacterial community structures in S. paramamosain. Hence, this study sought to examine gut microbial diversity and core gut microbiota of S. paramamosain from nine coastal regions in southern China. To the best of our knowledge, this study is the first to characterize core gut microbiota from S. paramamosain from southern Chinese coasts using state of the art, Illumina MiSeq sequencing methodologies.

Analysis of gut microbiota composition demonstrated that the dominant bacteria of the fifty-four samples belonged to six phyla, Proteobacteria, Firmicutes, Bacteroidetes, Tenericutes, Spirochaetae and Fusobacteria, and the first four phyla were also found in the Eriocheir sinensis gastrointestinal tract [29]. These results were consistent with a previous study on gut bacterial assemblages of Eriocheir sinensis from Lake Tai $(286 \mathrm{~km}$ from Lake Gucheng in China) [30]. These dominant genera may play major roles in gut function or adapt to the environment by the digestive tract.

The 11 core genera constituted a phylogenetic core of the genus, accounting for $48.81 \%$ of total sequences. Among them, Tenericutes from the genus Candidatus Hepatoplasma, accounted for the greatest average relative abundance. Previous research had discovered that isopods with intestinal tract based Candidatus_Hepatoplasma, had higher survival rates when food was deficient [31]. However, this has not yet been reported in S. paramamosain. In China, artificially cultured crabs are located in ponds, with little phytoplankton or zooplankton. Similarly, breeding densities are higher. In addition, farmers feed crabs at fixed times, therefore, $S$. paramamosain may be 


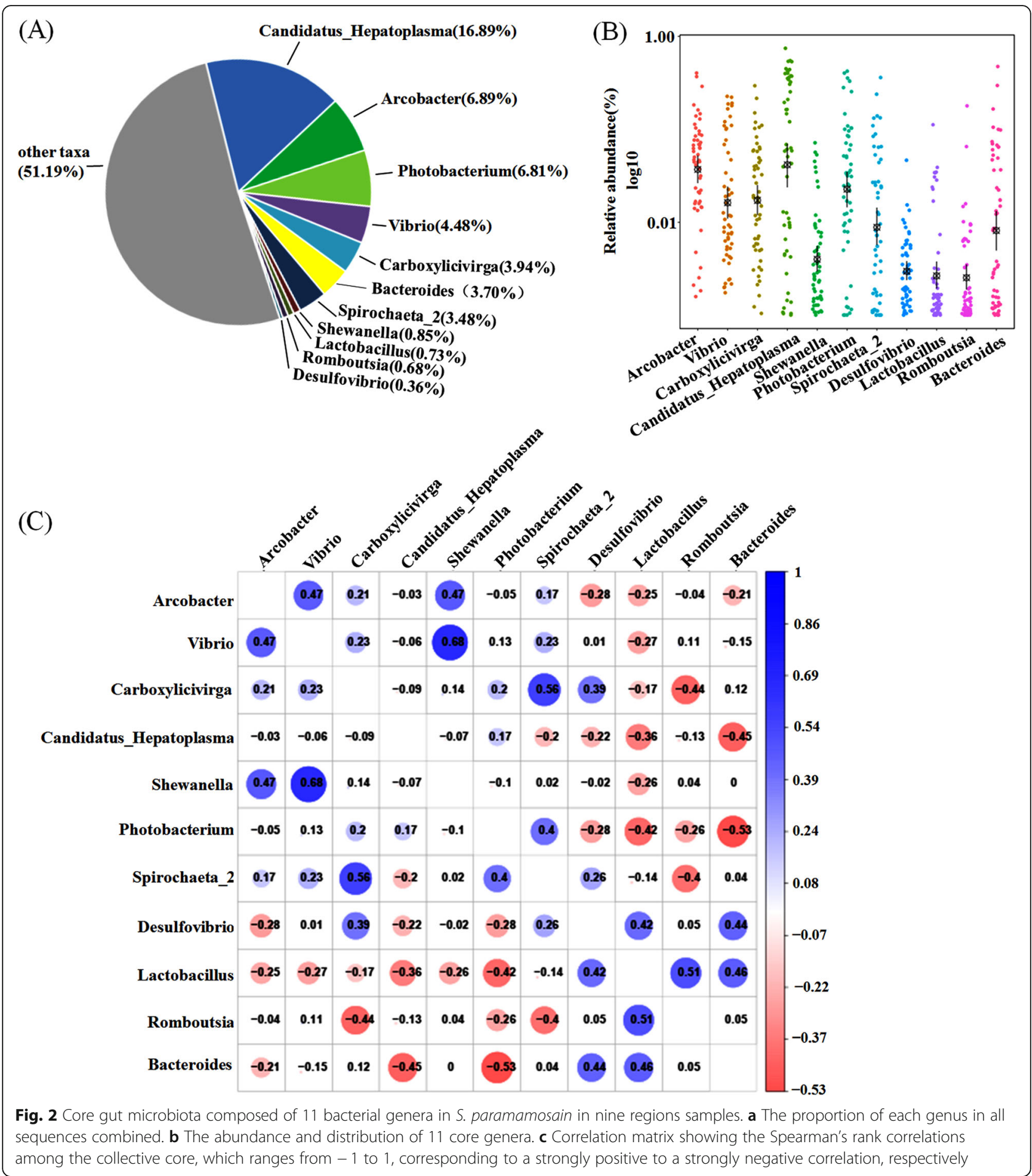

in hungry environments for prolonged periods. So, we speculated that the reason the mud crab could be able to adapt to thehunger environment is the regulation of Candidatus_Hepatoplasma. However, this conjecture must be corroborated by further research.

The core genera; Arcobacter, Photobacterium, Vibrio, Shewanella and Desulfovibrio belong to Proteobacteria.
The genus Arcobacter is common in many marine invertebrates, such as crabs [32], mussels [33], abalones [34], and oysters [35]. The genus Photobacterium, which is one of the nine genera in the family Vibrionaceae (order "Vibrionales", class Gammaproteobacteria), is the largest genera after Vibrio [36, 37]. Some of its species exhibit bioluminescence and pathogenesis mechanisms [38], 
Table 3 The core genera identified in female samples

\begin{tabular}{llll}
\hline Phylum & Genus & Relative abundance (\%) & Range (\%) \\
\hline Tenericutes & Candidatus_Hepatoplasma & 22.71 & $0.000-54.672$ \\
Proteobacteria & Photobacterium & 6.12 & $0.000-40.127$ \\
Proteobacteria & Arcobacter & 5.88 & $0.083-28.949$ \\
Bacteroidetes & Bacteroides & 5.55 & $0.000-47.259$ \\
Bacteroidetes & Carboxylicivirga & 4.78 & $0.206-29.438$ \\
Spirochaetae & Spirochaeta_2 & 4.54 & $0.000-35.887$ \\
Proteobacteria & Vibrio & 3.05 & $0.074-18.715$ \\
Firmicutes & Defluviitaleaceae_UCG_011 & $0.000-7.729$ \\
Firmicutes & Clostridium_sensu_stricto_1 & 1.59 & $0.000-4.331$ \\
Firmicutes & Clostridium_sensu_stricto_11 & 0.70 & $0.000-6.029$ \\
Firmicutes & Romboutsia & 0.52 & $0.000-6.419$ \\
Proteobacteria & Shewanella & 0.41 & $0.007-3.808$ \\
Firmicutes & Lactobacillus & 0.39 & $0.000-3.316$ \\
Proteobacteria & Desulfovibrio & 0.37 & $0.007-1.440$ \\
Tenericutes & Candidatus_Bacilloplasma & 0.29 & $0.000-1.511$ \\
\hline
\end{tabular}

with one study reporting that Photobacterium is a potential freshwater fish pathogen [39]. Worldwide, Vibrio is widely distributed in aquatic environments. However, many Vibrio members are considered primary pathogens in causing disease and death in aquaculture animals [40], and they seriously jeopardize the development of aquaculture. Many studies have shown that Vibrio provides a benefit to the host, for example, Asfie et al., [41] isolated multiple strains of Protease-producing bacteria from the gingiva intestinal tract, and showed that some proteases secreted by Vibrio are beneficial to gums, growth and development. Similarly, Hamid et al., [42] observed that Vibrio secreted amylases, proteases, lecithinases and chitinases to help digest important nutrients such as fat, proteins and carbohydrates in the host body. Further research also showed that Vibrio was present in both healthy and diseased S. paramamosain [27]. We therefore speculated that Vibrio may digest important nutrients such as fat, protein and carbohydrates in the host body by secreting amylases and proteases to maintain normal activities in healthy crabs. Therefore, it was not surprising that Vibrio was found in samples in this study. Our study has also illustrated the diversity of Vibrio and its beneficial role as a dominant bacteria in

Table 4 The core genera identified in male samples

\begin{tabular}{llll}
\hline Phylum & Genus & Relative abundance (\%) & Range (\%) \\
\hline Tenericutes & Candidatus_Hepatoplasma & 11.07 & $0.007-74.325$ \\
Proteobacteria & Arcobacter & 7.91 & $0.058-40.186$ \\
Proteobacteria & Photobacterium & 7.51 & $0.000-42.027$ \\
Proteobacteria & Vibrio & 5.90 & $0.098-22.604$ \\
Fusobacteria & Psychrilyobacter & 3.19 & $0.000-21.912$ \\
Bacteroidetes & Carboxylicivirga & 3.09 & $0.004-14.964$ \\
Spirochaetae & Spirochaeta_2 & 2.42 & $0.000-23.831$ \\
Bacteroidetes & Bacteroides & 1.84 & $0.000-16.469$ \\
Firmicutes & Defluviitaleaceae_UCG_011 & 1.47 & $0.000-12.943$ \\
Proteobacteria & Shewanella & 1.32 & $0.000-5.223$ \\
Firmicutes & Lactobacillus & 1.10 & $0.004-11.112$ \\
Firmicutes & Romboutsia & 0.94 & $0.000-17.830$ \\
Firmicutes & Lactococcus & 0.70 & $0.000-3.718$ \\
Firmicutes & Clostridium_sensu_stricto_1 & 0.60 & $0.000-4.932$ \\
Proteobacteria & Desulfovibrio & 0.43 & $0.000-4.543$ \\
\hline
\end{tabular}




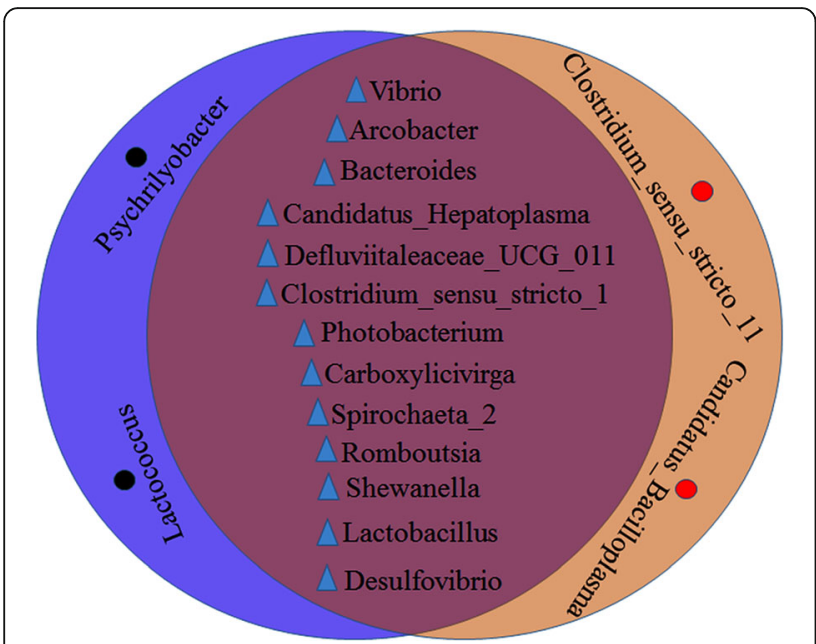

Fig. 3 Core genera identified in male and female samples

the intestine. The separation and identification of beneficial Vibrio species may promote crab aquaculture production.

Firmicutes are often found in the gut of marine invertebrates, such as sea squirt (Ciona intestinalis) [43], black tiger shrimp (Penaeus monodon) [44] and the Atlantic blue crab (Callinectes sapidus) [32]. The Lactobacillus genus belongs to the Firmicutes, which are commonly found in the gastrointestinal tracts of humans and other animals. In this study, Lactobacillus was also found in these nine regions, therefore, we speculated it may have potential probiotic properties in S. paramamosain. Studies have shown that due to its relevance in industrial applications in certain species, such as L. lactis, the central metabolic pathway of this genus has been extensively studied. These bacteria can convert large hexose sugar substrates to pyruvate via glycolysis and then to lactate [45]. Lactococcus is the focus of intensive research in carbohydrate catabolism, the industrial fermentation process [46] and its role in promoting health, such as the prevention and protection of diarrhea and intestinal infections, are important for a well-balanced gut microbiota [47, 48]. So, on one hand, due to it can prevent and protect diarrhea and intestinal infections, it is not surprising that it can be found in all samples from nine regions. On the other hand, it is worthy of further study to isolate and characterize the functional bacteria of this genus from intestinal samples, and it may develop probiotics for the S. paramamosain breeding industries. Moreover, the genus Bacteroides from the Bacteroidetes phyla has been associated with animal protein metabolism, a variety of 354 Yang et al., amino acids and saturated fats [49]. And other core genera of Shewanella, Desulfovibrio, Romboutsia, Carboxylicivirga and Spirochaeta_2 in functional study have not yet been reported.

According to the experience of many farmers, there were differences in development processes between male and female crabs. However, there were 13 identical genera in the mid-gut population of male and female crabs (Fig. 3). This meant that gender had no significant effects on gut composition in S. paramamosain. This was consistent with Jin et al., [50] and their study, the intestinal flora of Eriocheir sinensis in Yangcheng West Lake.

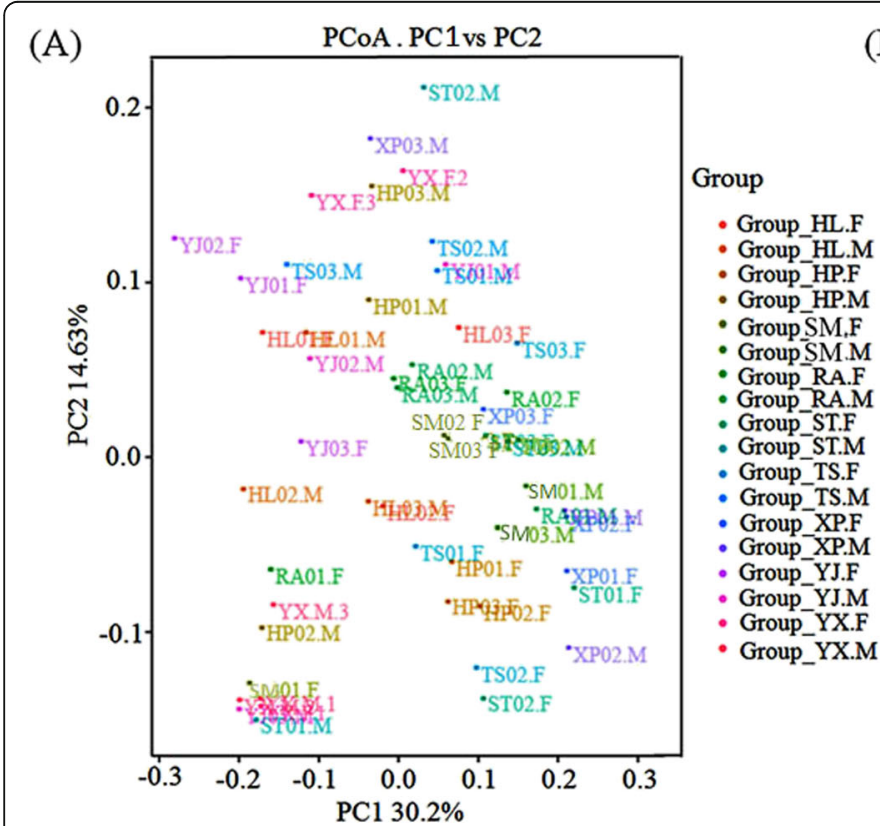

(B)

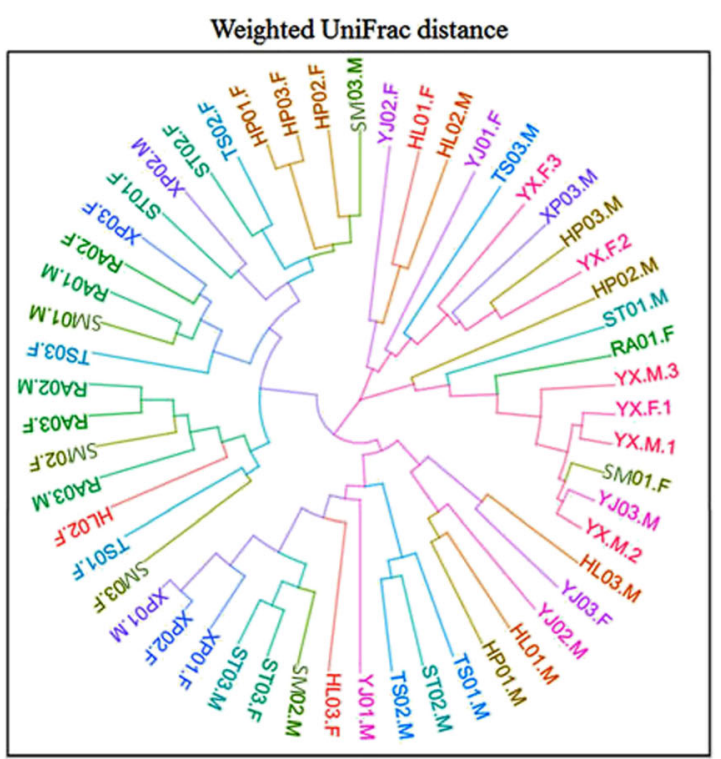

Fig. 4 Principal coordinate analysis and circular tree plot of all samples using the weighted UniFrac distance matrices. a Principal coordinate analysis of the microbial communities in all samples; $\mathbf{b}$ Circular tree plot of all samples using the weighted UniFrac distance matrices 

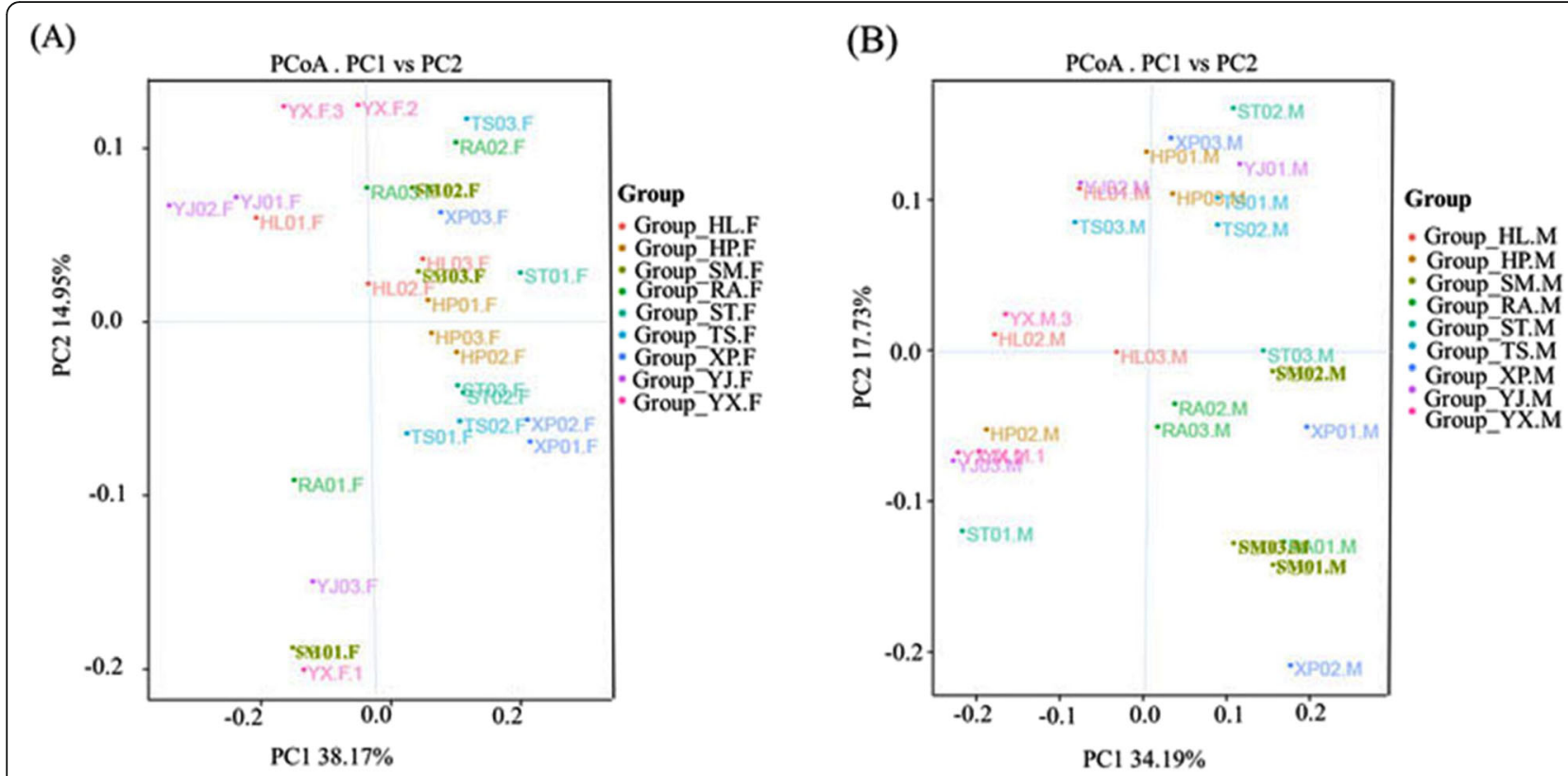

(C)

Weighted UniFrac distance

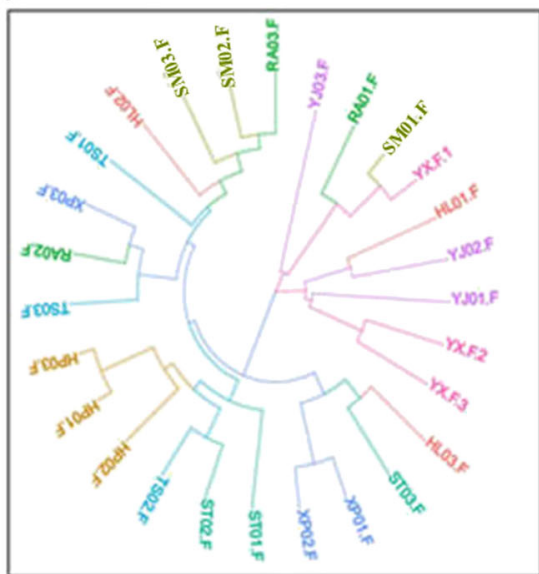

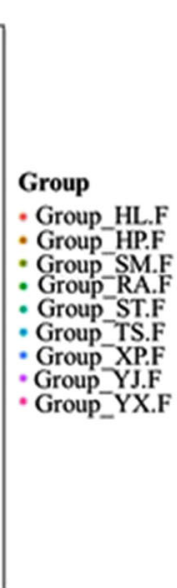

(D)

Weighted UniFrac distance

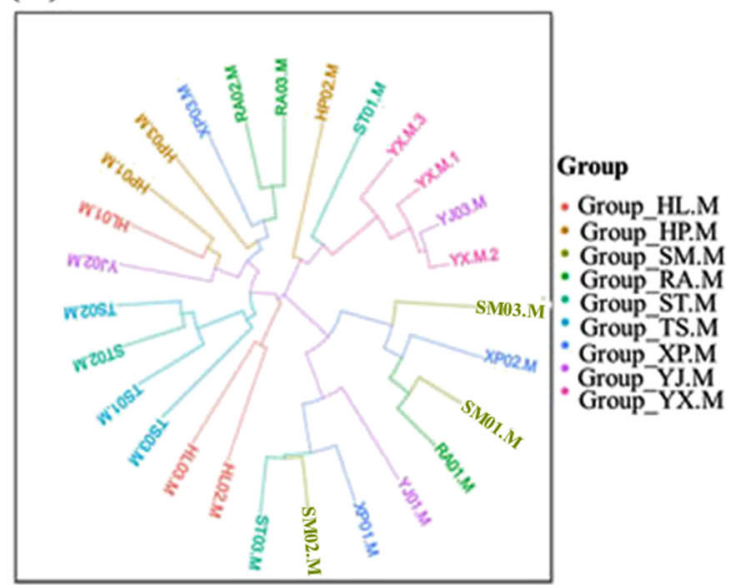

Fig. 5 Principal coordinate analysis and circular tree plot of female or male samples using the weighted UniFrac distance matrices. a Principal coordinate analysis of the microbial communities in female samples; $\mathbf{b}$ Principal coordinate analysis of the microbial communities in male samples; c Circular tree plot of female samples using the weighted UniFrac distance matrices; $\mathbf{d}$ Circular tree plot of male samples using the weighted UniFrac distance matrices

An interesting discovery from this study was the difference between male and female samples in terms of the core gut microbiota, at the genus level. There were two different core genera in female samples (Clostridium sensu_stricto_11 and Candidatus_Bacilloplasma), and two additional core genera in male samples (Psychrilyobacter and Lactococcus). At present, the functions of these four genera have not been reported. We speculated these genera may be the reason for differences in flavors between male and female crabs. It will be interesting to determine which gut microbiota cause flavor differences. In this study, the 11 core genera were distributed in the five dominant phyla. Among these, five genera
(Arcobacter, Photobacterium, Vibrio, Shewanella, Desulfovibrio) belonged to Proteobacteria, while the other four phyla contained one or two genera, such as Bacteroidetes (genus Carboxylicivirga \& Bacteroides), Firmicutes (genus Lactobacillus \& Romboutsia), Tenericutes (genus Candidatus_Hepatoplasma), and Spirochaetae (genus Spirochaeta_2). We speculated that these phyla and genera present in the $S$. paramamosain' gut might have many reasons. Firstly, in terms of internal factors, in China, although the seedlings of these S. paramamosain have broken through the key technologies of large-scale breeding [51], the artificial breeding technology is not quite ripe yet. So mud crab seedlings 
are mainly derived from natural sea areas, these crabs in nine regions may be not the same source, they are the same species, S. paramamosain, therefore they are hereditary. We suspect that they have these common core genera that may be genetic factors.

On the other hand, environmental factors have also been reported to affect gut microbial composition [52]. Our analyses found 11 core genera in nine different regions. This observation may indicate that environmental factors do little to affect the structure of the gut microbiota of S. paramamosain or the lack of an effect may have been due to similar natural conditions (Additional file 1: Table S4), such as temperature, salinity, $\mathrm{pH}$, dissolved oxygen (DO) and so on. It may be that these nine regions are all at suitable temperatures and salinities and they live in ponds that are separated by mud with black plastic membranes to prevent the loss of crabs.

\section{Conclusions}

In this study, we used Illumina MiSeq sequencing to clarify the gut microbiota of composition from S. paramamosain. This was the first time we identified core intestinal microbiota from nine coastal regions of southern China. We analyzed 472,782 valid tags from nine regions samples, of which 2552 OTUs were identified. Our results showed that Proteobacteria, Firmicutes, Bacteroidetes, Tenericutes, Spirochaetae and Fusobacteria were the dominant phyla of the 36 representative phyla. 11 genera of the 820 representative genera were considered as core gut microbiota and were distributed in the five dominant phyla. The core genera distributed in Proteobacteria were the most genera, including Arcobacter, Photobacterium, Vibrio, Shewanella, Desulfovibrio. While other four phyla contain one or two genera. Moreover, there were differences between male and female samples in the core gut microbiota, at the genus level. There were two special genera in female samples (Clostridium sensu_stricto_11 and Candidatus_Bacilloplasma), and two special genera in male samples (Psychrilyobacter and Lactococcus). This study has generated much sequencing data related to the gut microbiota of $S$. paramamosain and may contribute to probiotic development for mud crab aquaculture industries in the future.

\section{Methods}

\section{Sample collection in different areas}

Mud crab breeding is mainly distributed along the coast of southern China (Additional file 1: Figure S5), including Zhejiang, Fujian, Guangdong, Guangxi, Hainan provinces. Firstly, we purchased crabs from local farmers in nine areas between May and June 2017. These crab collection areas were at Sanmen county, Taizhou city, Zhejiang province (SM, 29 $\left.06^{\prime} 16.81^{\prime \prime} \mathrm{N}, 121^{\circ} 23^{\prime} 44.45 \mathrm{E}\right)$, Ruian county, Wenzhou city, Zhejiang province (RA, $27^{\circ} 46^{\prime} 42.17^{\prime \prime} \mathrm{N}, 120^{\circ} 39^{\prime}$
18.65 "E), Xiapu county, Ningde city, Fujian province (XP, $26^{\circ} 53^{\prime} 6.61^{\prime \prime} \mathrm{N}, 120^{\circ} 0^{\prime} 20.02^{\prime \prime} \mathrm{E}$ ), Yunxiao county, Zhangzhou city, Fujian province (YX, 235' $\left.29.02^{\prime \prime} \mathrm{N}, 117^{\circ} 20^{\prime} 22.744^{\prime \prime} \mathrm{E}\right)$, Shantou city, Guangdong province (ST, 23⒉'22.19"N, $\left.116^{\circ} 40^{\prime} 39.89^{\prime \prime} \mathrm{E}\right)$, Yangjiang city, Guangdong province (YJ, $\left.21^{\circ} 51^{\prime} 39.78^{\prime \prime} \mathrm{N}, 111^{\circ} 58^{\prime} 39.04^{\prime \prime} \mathrm{E}\right)$, Taishan county, Zhuhai city, Guangdong province (TS, $22^{\circ} 12^{\prime} 24.13^{\prime \prime} \mathrm{N}, 113^{\circ} 17^{\prime}$ $50.43^{\prime \prime E)}$, Hepu county, Behai city, Guangxi zhuang autonomous region (HP, 21 $\left.39^{\prime} 48.50^{\prime \prime} \mathrm{N}, 109^{\circ} 12^{\prime} 10.98^{\prime \prime} \mathrm{E}\right)$, Hele county, Wanning city, Hainan province (HL, $18^{\circ} 54^{\prime}$ $\left.0.52^{\prime \prime} \mathrm{N}, 110^{\circ} 28^{\prime} 32.39^{\prime \prime} \mathrm{E}\right)$. In these locations, we caught mud crab. Each region chose good limbs and good vitality 12 female and male crabs (body weight: $250-450 \mathrm{~g}$ ), immediately isolated their intestinal tracts and contents and froze samples in liquid nitrogen. Samples were transported to the lab on dry ice. When extracting DNA, every three samples were mixed into one, so each region had six samples, with a total of 54 . All samples were stored in sterile containers.

\section{DNA extraction, 16S rRNA gene amplification, and Illumina MiSeq sequencing}

Genomic DNA was extracted from each sample using the DNeasy PowerLyzer PowerSoil Kit (QIAGEN, Hilden, Germany). DNA concentration and purity were assessed on $1 \%$ agarose gels. The V3-V4 region of the bacterial $16 \mathrm{~S}$ rRNA gene was amplified using the barcode-fusion forward primer $343 \mathrm{~F}$ (5'- TACGGRAGGCAGCAG-3') and the reverse primer 798R (5' - AGGGTATCTAATCCT $\left.3^{\prime}\right)$. The PCR was performed in a $30 \mu$ l volume with $15 \mu \mathrm{l}$ of $2 \times \mathrm{HiFi}$ Hot Start Ready Mix, $1.0 \mu \mathrm{l}$ of forward and reverse primers $(10 \mu \mathrm{mol} / \mathrm{L})$, and $50 \mathrm{ng}$ of genomic DNA as template. Thermal cycling consisted of initial denaturation at $94{ }^{\circ} \mathrm{C}$ for $5 \mathrm{~min}$; followed by 26 cycles of denaturation at $94{ }^{\circ} \mathrm{C}$ for $30 \mathrm{~s}$, annealing at $56^{\circ} \mathrm{C}$ for $30 \mathrm{~s}$, elongation at $72{ }^{\circ} \mathrm{C}$ for $30 \mathrm{~s}$ and a final extension at $72{ }^{\circ} \mathrm{C}$ for $7 \mathrm{~min}$.

The PCR products were purified using Agencourt Ampure XP beads (Beckman, CA, USA) according to the manufacturer's instructions. $5 \mu \mathrm{l}$ purified product were detected by $1 \%$ agarose gel electrophoresis and $1 \mathrm{ul}$ purified product for concentration detection using a NanoDrop Lite spectrophotometer (Thermo Scientific). The purified DNA was then used as a template to perform a second PCR amplification using the same primer sequences and the protocol described above, however, 8 cycles were employed. After a further purification with the AMPure XP beads, the amplicon was measured concentration using NanoDrop Lite spectrophotometer. Purifed amplicons were pooled in equimolar and pairedend sequenced $(2 \times 300)$ on an Illumina MiSeq platform.

\section{Bioinformatic analyses}

Raw sequencing data came in FASTQ format. Pairedend reads were preprocessed using Trimmomatic 
software [53], to detect and remove ambiguous bases $(\mathrm{N})$. The software also removed low quality sequences, with average quality scores below 20, using the sliding window trimming approach. After trimming, paired-end reads were assembled using FLASH software [54]. The parameters for assembly were: $10 \mathrm{bp}$ minimal overlap, $200 \mathrm{bp}$ maximum overlap and a $20 \%$ maximum mismatch rate. Sequences were performed further denoising as follows: contigs with ambiguous, homologous sequences or below $200 \mathrm{bp}$ were abandoned. Reads with $75 \%$ of bases above Q20 were retained. Contigs with chimeras were detected and removed. These steps were achieved using the QIIME software (version 1.8.0) [55]. Clean reads were subjected to primer sequence removal and clustering to generate operational taxonomic units (OTUs) using UPARSE software, with 97\% similarity cutoff [56]. The representative read of each OTU was selected using QIIME software. All representative reads were annotated and blasted against the Silva database Version 123 (or Greengens) (16 s rDNA) using RDP classifier (confidence threshold was 70\%) [57]. For alpha-diversity metrics, Observed Species, Chao1 estimator, Shannon Wiener Index, Simpson diversity index, Good's Coverage with QIIME (Version 1.8.0) and displayed with R software (Version 2.15.3). For beta-diversity metrics, the weighted UniFrac distance matrix [58] was calculated and visualized using Principal Coordinate Analysis (PCoA) in QIIME. Biomarker discovery analysis of each taxonomic unit was performed using LeFse (version 1.0.7) [59]. All figures were generated with customized R scripts.

\section{Statistics}

All statistical analyses were performed using SPSS (SPSS 21.0). ANOVA was also used for statistical analyses. Correlations between the core genera of gut microbiota were appraised by calculating nonparametric Spearman's rank correlation coefficients, which were displayed in a correlation matrix. Results were considered significant at $P<0.05$.

\section{Supplementary information}

Supplementary information accompanies this paper at https://doi.org/10. 1186/s12864-019-6219-7.

Additional file 1: Table S1. Number of bacterial taxonomic units. Table S2. Mean relative abundance of the 15 most abundant phyla in samples from the nine coastal regions of Southern China. Table S3. Mean relative abundance of the 15 most abundant genera in samples from the nine coastal regions of Southern China. Table S4. Salinity, temperature, $\mathrm{pH}$, dissolved oxygen (DO), ammonia-nitrogen and nitrite levels of the nine regions of Southern China. Figure S1. Impact of sequencing depth and sampling on bacterial phylotypes. Figure S2. Composition of microbial communities at the Class level. Figure S3. Composition of microbial communities at the Order level. Figure $\mathbf{5 4}$. Composition of microbial communities at the Family level. Figure $\mathbf{S 5}$. Sample collection in the nine coastal regions of Southern China.

\section{Abbreviations}

F: female; HL: Hele county, Wanning city, Hainan province; HP: Hepu county, Behai city, Guangxi zhuang autonomous region; M: male; RA: Ruian county, Wenzhou city, Zhejiang province; S. paramamosain: Scylla paramamosain; SM: Sanmen county, Taizhou city, Zhejiang province; ST: Shantou city, Guangdong province; TS: Taishan county, Zhuhai city, Guangdong province; XP: Xiapu county, Ningde city, Fujian province; YJ: Yangjiang city,

Guangdong province; YX: Yunxiao county, Zhangzhou city, Fujian province

\section{Acknowledgements}

We thank Oebiotech (Oebiotech, Shanghai, China) for sequencing consultation and support.

\section{Authors' contributions}

CW and HW1 (Huan Wang) conceived and designed the study. HW2 (Hongling Wei), HW1 and LT took samples of experimental animals. HW2, $H W 1, L T, C M, C Y$ and LC performed and analyzed all the other experiments. HW2 wrote the manuscript with support from all authors. All authors read and approved the final manuscript.

\section{Funding}

This work was supported by the Major Science and Technology Special Project of Zhejiang Province (No. 2016C02055-8); the Ministry of Agriculture of China and China Agriculture Research System (No. CARS-48); the Marine and Fishery Bureau, Sanmen County, Zhejiang Province (HK2017000088); and the K. C. Wong Magna Fund in Ningbo University. The funders had no role in the study design, data collection and analysis, decision to publish, or preparation of the manuscript.

\section{Availability of data and materials}

Raw Illumina sequences were deposited in the National Center for Biotechnology Information (NCBI) and our SRA records will be accessible with the following link after the indicated release date: https://www.ncbi. nlm.nih.gov/sra/ SRP162721, SRA accession numbers: SRP162721.

\section{Ethics approval and consent to participate}

The animals used in this study are crabs, which are invertebrates and are exempt from ethical requirements. S. paramamosain is not an endangered or protected species. All animal work was conducted according to the relevant national and international guidelines. No specific permissions are required to work with invertebrates in China. Similarly, no specific permissions are required for the collection of S. paramamosain from sample sites because they were not collected from protected areas.

\section{Consent for publication}

Not applicable.

\section{Competing interests}

The authors declare that they have no competing interests.

\section{Author details}

'School of Marine Science, Ningbo University, Ningbo 315211, Zhejiang, China. ${ }^{2}$ Key Laboratory of Applied Marine Biotechnology, Ministry of Education, Ningbo University, Ningbo 315211, Zhejiang, China. ${ }^{3}$ Agricultural and Rural Bureau, Sanmen County, Zhejiang Province, China. ${ }^{4}$ Fishery Technology Station, Sanmen County, Zhejiang Province, China.

Received: 20 December 2018 Accepted: 23 October 2019 Published online: 08 November 2019

\section{References}

1. Lin Z, Hao M, Zhu D, et al. Molecular cloning, mRNA expression and nutritional regulation of a $\Delta 6$ fatty acyl desaturase-like gene of mud crab, Scylla paramamosain. Comp Biochem Physiol B Biochem Mol Biol. 2017;208-209:29-37.

2. Walton ME, Vay LL, Lebata JH, et al. Seasonal abundance, distribution and recruitment of mud crabs (Scylla, spp.) in replanted mangroves. Estuar Coast Shelf Sci. 2006;66(3-4):493-500.

3. Wang $\mathrm{H}$, Wei $\mathrm{H}$, Tang $\mathrm{L}$, et al. A proteomics of gills approach to understanding salinity adaptation of Scylla paramamosain. Gene. 2018a;677:119-31.

4. Wang Z, Sun B, Zhu F. Epigallocatechin-3-gallate inhibit replication of white spot syndrome virus in Scylla paramamosain. Fish Shellfish Immunol. 2017:67:612-9.

5. China fishery statistical yearbook, China's Ministry of Agriculture, 2018. 
6. Ma LB, Zhang FY, Ma CY, et al. Scylla paramamosain (Estampador) the most common mud crab (Genus Scylla) in China: evidence from mtDNA. Aquac Res. 2006;37:1694-8.

7. Wang $\mathrm{H}$, Wei $\mathrm{H}$, Tang $\mathrm{L}$, et al. Identification and characterization of miRNAs in the gills of the mud crab (Scylla paramamosain) in response to a sudden drop in salinity. BMC Genomics. 2018b;19:609.

8. Ye H, Tao Y, Wang GZ, et al. Experimental nursery culture of the mud crab Scylla paramamosain (Estampador) in China. Aquacult Int. 2011;19:313-21.

9. Greathouse KL, Faucher MA, Hastings-Tolsma M. The gut microbiome, obesity, and weight control in Women's reproductive health. West I Nurs Res. 2017;39(8):1094-119.

10. Kau AL, Ahern PP, Griffin NW, et al. Human nutrition, the gut microbiome and the immune system. Nature. 2011:474(7351):327-36.

11. Huang CH, Yu X, Liao WB. The Expensive-Tissue Hypothesis in Vertebrates: Gut Microbiota Effect, a Review. Int J Mol Sci. 2018;19(6):1792.

12. Rizzetto L, Fava F, Tuohy KM, et al. Connecting the immune system, systemic chronic inflammation and the gut microbiome: the role of sex. J Autoimmun. 2018;92:12-34.

13. Ok SJ. Gut microbiota in inflammatory bowel disease. Pediatr Gastroenterol Hepatol Nutr. 2013;16(1):17-21.

14. Qin N, Yang F, Li A, et al. Alterations of the human gut microbiome in liver cirrhosis. Nature. 2014;513(7516):59-64.

15. Garrett WS. Cancer and the microbiota. Science. 2015;348:80-6.

16. Meijnikman AS, Gerdes VE, Nieuwdorp M, et al. Evaluating causality of gut microbiota in obesity and diabetes in humans. Endocr Rev. 2018;39(2):133-53.

17. Han H, Li Y, Fang J, et al. Gut Microbiota and Type 1 Diabetes. Int J Mol Sci. 2018;19(4):995

18. Cheung MK, Yip HY, Nong W, et al. Rapid change of microbiota diversity in the gut but not the Hepatopancreas during gonadal development of the new shrimp model Neocaridina denticulata. Mar Biotechnol (NY). 2015;17(6):811-9.

19. Olmos J, Ochoa L, Paniagua-Michel J, et al. Functional feed assessment on Litopenaeus vannamei using 100\% fish meal replacement by soybean meal high levels of complex carbohydrates and Bacillus probiotic strains. Mar Drugs. 2011;9(6):1119-32

20. Wang $Y$, Zhang C, Wang H, et al. Involvement of a newly identified atypical type II crustin (SpCrus5) in the antibacterial immunity of mud crab Scylla paramamosain. Fish Shellfish Immunol. 2018:75:346-56.

21. Xiong J. Progress in the gut microbiota in exploring shrimp disease pathogenesis and incidence. Appl Microbiol Biotechnol. 2018;102(17): 7343-50.

22. Wu T, Zhang Z, Cai C, et al. The effect of pectin and Xylan on intestinal microflora structure of Chinese mitten crab. Genomics \& Applied Biology. 2015;34(4):745-53 (In Chinese with English abstract).

23. Zhang ML, Sun YH, Chen LQ, et al. Symbiotic bacteria in gills and guts of Chinese mitten crab (Eriocheir sinensis) differ from the free-living bacteria in water. PLoS One. 2016;11(1):e0148135.

24. Waite DW, Eason DK, Taylor MW. Influence of hand rearing and bird age on the fecal microbiota of the critically endangered kakapo. Appl Environ Microbiol. 2014;80(15):4650-8.

25. Qin J, Li R, Raes J, et al. A human gut microbial gene catalogue established by metagenomic sequencing. Nature. 2010;464(7285):59-65.

26. Roeselers G, Mittge EK, Stephens WZ, et al. Evidence for a core gut microbiota in the zebrafish. ISME J. 2011;5(10):1595-608.

27. Li S, Sun L, Wu H, et al. The intestinal microbial diversity in mud crab (Scylla paramamosain) as determined by PCR-DGGE and clone library analysis. J Appl Microbiol. 2012;113(6):1341-51.

28. Pajarillo EA, Ju KO, Chae JP, et al. Administration of probiotic Enterococcus faecium NCIMB 11181 changes the fecal microbiota and diversity in weaned pigs. Asian Conference on Lactic Acid Bacteria. 2015.

29. Ding ZF, Cao MJ, Zhu XS, et al. Changes in the gut microbiome of the Chinese mitten crab (Eriocheir sinensis) in response to white spot syndrome virus (WSSV) infection. J Fish Dis. 2017;40(11):1561-71.

30. Chen $X$, Di P, Wang $H$, et al. Bacterial community associated with the intestinal tract of Chinese mitten crab (Eriocheir sinensis) farmed in Lake tai. China PLoS One. 2015;10(4):e0123990.

31. Fraune S, Zimmer M. Host-specificity of environmentally transmitted mycoplasma-like isopod symbionts. Environ Microbiol. 2008:10(10):2497-504

32. Givens $C E$, Burnett $K G$, Burnett $L E$, et al. Microbial communities of the carapace, gut, and hemolymph of the Atlantic blue crab, Callinectes sapidus Mar Biol, 2013; 160 (11):2841-2851.
33. Collado L, Cleenwerck I, Van TS, et al. Arcobacter mytili sp. nov., an indoxyl acetate-hydrolysis-negative bacterium isolated from mussels. Int J Syst Evol Microbiol. 2009;59(Pt 6):1391-6.

34. Tanaka R, Ootsubo M, Sawabe T, et al. Biodiversity and in situ abundance of gut microflora of abalone (Haliotis discus hannai) determined by cultureindependent techniques. Aquaculture. 2004;241(1):453-63.

35. Romero J, Garcíavarela M, Laclette JP, et al. Bacterial 16s rRNA gene analysis revealed that bacteria related to Arcobacter spp. constitute an abundant and common component of the oyster microbiota (Tiostrea chilensis). Microb Ecol. 2002;44(4):365-71.

36. Pérez-Cataluña A, Lucena T, Tarazona E, et al. An MLSA approach for the taxonomic update of the Splendidus clade, a lineage containing several fish and shellfish pathogenic Vibrio spp. Syst Appl Microbiol. 2016;39(6):361-9.

37. Sawabe T, Ogura Y, Matsumura Y, et al. Updating the Vibrio clades defned by multilocus sequence phylogeny: Proposal of eight new clades, and the description of Vibrio tritonius sp. nov. Front Microbiol. 2013;4:414.

38. Labella AM, Arahal DR, Castro D, et al. Revisiting the genus Photobacterium: taxonomy, ecology and pathogenesis. Int Microbiol. 2017;20(1):1-10.

39. Cantas L, Sørby JR, Aleström $P$, et al. Culturable gut microbiota diversity in Zebrafish. Zebrafish. 2012;9(1):26-37.

40. Wang $\mathrm{Fl}$, Chen JC. The immune response of tiger shrimp Penaeus monodon, and its susceptibility to Photobacterium damselae, subsp. damselae, under temperature stress. Aquaculture. 2006;258(1):34-41.

41. Hansen GH, Olafsen JA. Bacterial interactions in early life stages of marine cold water fish. Microb Ecol. 1999;38(1):1-26.

42. Hamid A, Sakata T, Kakimoto D. Microflora in the alimentary tract of grey mullet. IV. Estimation of enzyme activities of the intestinal bacteria. Nihonsuisan-gakkai-shi. 1979;45(1):99-106.

43. Dishaw $L$, Florestorres J, Lax S, et al. The gut of geographically disparate Ciona intestinalis harbors a core microbiota. PLoS One. 2014;9(4):e93386.

44. Rungrassamee W, Klanchui A, Maibunkaew S, et al. Characterization of intestinal bacteria in wild and domesticated adult black tiger shrimp (Penaeus monodon). PLoS One. 2014;9(3):e91853.

45. Price $C E$, Zeyniyev A, Kuipers OP, et al. From meadows to milk to mucosaadaptation of Streptococcus, and Lactococcus, species to their nutritional environments. FEMS Microbiol Rev. 2012;36(5):949-71.

46. Mayo B, Aleksandrzak-Piekarczyk T, Fernández $M$, et al. Updates in the metabolism of lactic acid bacteria. In: Mozzi F, Raya RR, Vignolo GM, editors. Biotechnology of lactic acid bacteria: novel applications. Oxford, U.K.: Wiley-Blackwell; 2010. p. 3-33. https://doi.org/10.1002/ 9780813820866.ch1.

47. Konstantinov SR. Lactobacilli in the porcine intestine: From composition to functionality. Wageningen, the Netherlands: PhD Thesis Wageningen University; 2005

48. Hu Y, Dun Y, Li S, et al. Dietary Enterococcus faecalis LAB31 improves growth performance, reduces diarrhea, and increases fecal Lactobacillus number of weaned piglets. PLoS One. 2015:10(1):e0116635.

49. Zhang J, Guo Z, Lim AQ, et al. Mongolians core gut microbiota and its correlation with seasonal dietary changes. Sci Rep. 2014;4:5001.

50. Jin B. Study on the intestinal flora of Eriocheir sinensis in Yangcheng West Lake. Nanjing Agricultural University, 2016.

51. Qiao ZG, Ma LB, Yu ZL, et al. Current status and development goals of marine crab culture in China. Fisheries Modernization. 2009:36(3):45-8 (In Chinese).

52. Bian GR, Ma SQ, Zhu ZG, et al. Age, introduction of solid feed and weaning are more important determinants of gut bacterial succession in piglets than breed and nursing mother as revealed by a reciprocal cross-fostering model. Environ Microbiol. 2016;18(5):1566-77.

53. Bolger AM, Lohse M, Usadel B. Trimmomatic: a flexible trimmer for Illumina sequence data. Bioinformatics. 2014;30(15):2114-20.

54. Reyon D, Tsai SQ, Khayter C, et al. FLASH assembly of TALENs for highthroughput genome editing. Nat Biotechnol. 2012;30(5):460-5.

55. Caporaso JG, Kuczynski J, Stombaugh J, et al. QIIME allows analysis of high-throughput community sequencing data. Nat Methods. 2010;7(5): 335-6.

56. Edgar RC. UPARSE: highly accurate OTU sequences from microbial amplicon reads. Nat Methods. 2013;10(10):996-8.

57. Wang Q, Garrity GM, Tiedje JM, et al. Naive Bayesian classifier for rapid assignment of rRNA sequences into the new bacterial taxonomy. Appl Environ Microbiol. 2007;73(16):5261-7. 
58. Lozupone C, Knight R. UniFrac: a new phylogenetic method for comparing microbial communities. Appl Environ Microbiol. 2005;71:8228-35.

59. Segata $\mathrm{N}$, Izard J, Waldron $\mathrm{L}$, et al. Metagenomic biomarker discovery and explanation. Genome Biol. 2011;12(6):R60.

\section{Publisher's Note}

Springer Nature remains neutral with regard to jurisdictional claims in published maps and institutional affiliations.

Ready to submit your research? Choose BMC and benefit from:

- fast, convenient online submission

- thorough peer review by experienced researchers in your field

- rapid publication on acceptance

- support for research data, including large and complex data types

- gold Open Access which fosters wider collaboration and increased citations

- maximum visibility for your research: over $100 \mathrm{M}$ website views per year

At $\mathrm{BMC}$, research is always in progress.

Learn more biomedcentral.com/submissions 